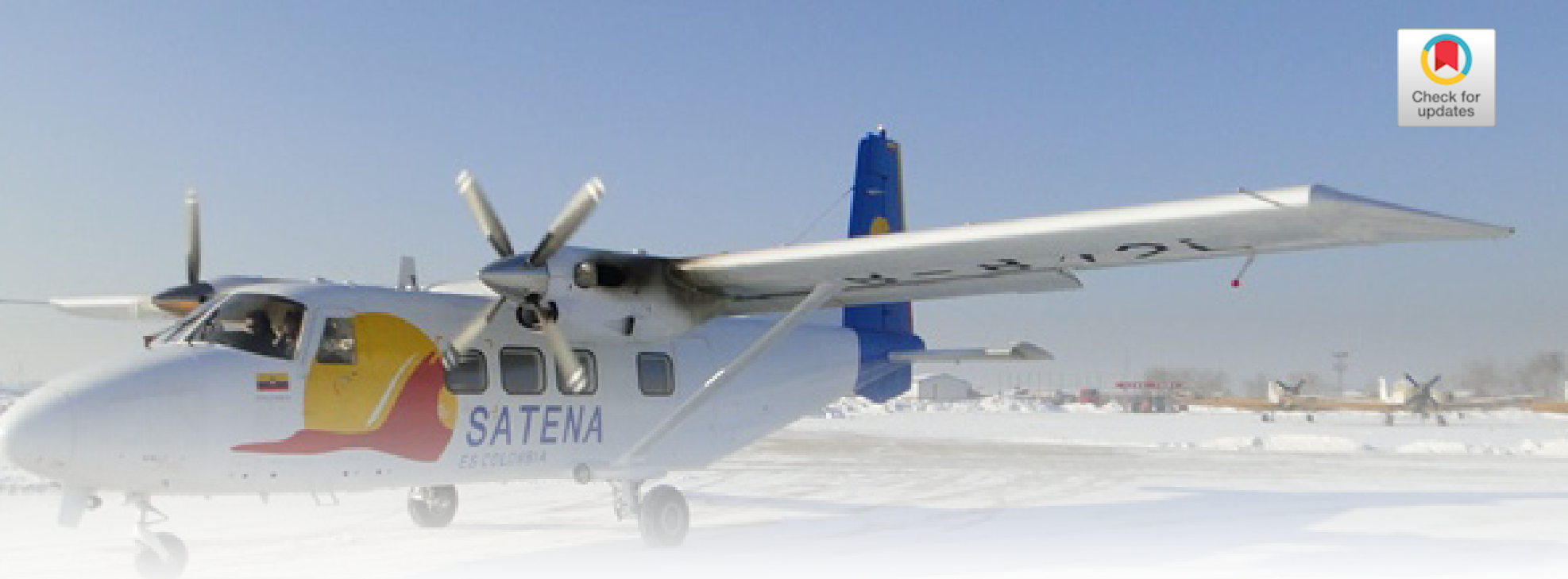

\title{
La ciberguerra china desde la lógica de la guerra irrestricta
}

\author{
Chinese Cyberwarfare from the Logic of the Unrestricted Warfare ${ }^{2}$
}

José Mauricio Mancera ${ }^{3}$

CIENCIA Y PODER AÉREO

ISSN 1909-7050 | E-ISSN 2389-2468 |Volumen 9 | Enero-Diciembre de 2014 | Colombia | Pp. 89-96

Recibido: 11/04/2014

Aprobado evaluador interno: 10/10/2014

Aprobado evaluador externo: 08/11/2014

Artículo de reflexión derivado de debates y puntos de reflexiones académicas en curso de ascenso.

${ }^{2}$ Reflection article from debates and academic reflections during the course for military promotion.

${ }^{3}$ Brigadier General del Aire. Fuerza Aérea Colombiana. Magíster en Estudios de Seguridad y Defensa de las Américas, Academia Nacional de Estudios Políticos y Estratégicos de Chile. Correo electrónico: mauromancera@hotmail.com

General Fuerza Aérea Colombiana. Master in Studies of Security and Defense of the Americas, Chilean National Academy for Political and Strategic Studies. E-mail:mauromancera@hotmail. com
Resumen: El presente artículo se orienta a demostrar la notoria intención de China en el sentido de querer disputar la hegemonía global a los Estados Unidos, utilizando una doctrina en particular, Ilamada la Guerra Irrestricta, con una herramienta específica designada como la ciberguerra, comprendida como: nueva, productiva, discreta y poco comprometedora forma de lucha, en defensa de intereses nacionales, en la mayoría de los casos, contra un enemigo asimétrico y con un poder mucho mayor, como son los Estados Unidos. Las evidencias, hechos y datos presentados, permitirán comprender tales intenciones e intereses por parte de China.

\section{Palabras clave: Ciberespacio, ciberguerra, guerra irrestricta, informática.}

Abstract: This article is oriented to demonstrate the notorious Chinese intention in the sense of wishing to dispute the global hegemony to the United States, using a particular doctrine, called the unrestricted warfare, with an specific designated tool as the cyberwarfare, understood as: new, productive, discrete and fairly compromise way of fighting, in defense of national interest, in most of the cases, against an asymmetric enemy and with a much greater power, as the United States are. The evidences, facts, and data presented, will allow understanding such intentions and interests of China.

Key Words: Computing, Cyberspace, Cyberwarfare, Unrestricted Warfare. 


\section{Introducción}

Acontecimientos y publicaciones dejan entre ver que la utilización de todas las formas de lucha por parte del Ejército de Liberación del Pueblo (PLA) ${ }^{4}$ y de otras instancias de la nación China, son un imperativo en la actualidad y quizás la ciberguerra es una de las principales expresiones de tal beligerancia soterrada, utilizada por China frente al gran poder norteamericano. Las evidencias de la participación de dicha nación en ataques cibernéticos y otra serie de acciones no se han podido materializar del todo, pero lo consignado en documentos como la Política Nacional de Defensa China, (República Popular China, 2010) en los libros "La Guerra Irrestricta"5 (Liang y Xiangsui, 1999) O "El Siglo de la China" (Shenkar, 2004) entre otras publicaciones; así como lo acontecido en la Operación Aurora, en la que se configura el robo de propiedad intelectual a compañías norteamericanas (Shakarian, Shakarian, y Ruef, 2013a), son realidades que conllevan a pensar en el uso masivo de formas no convencionales de guerra, donde la única regla es que no existen reglas, predominando la asimetría y el uso de espacios diferentes al físico, entre otras características.

En este orden de ideas, este artículo busca hacer una serie de planteamientos con los que se intenta demostrar el uso de una guerra sin límites o Guerra Irrestricta por parte de China, con un énfasis especial en la ciberguerra como: nueva, productiva, discreta y poco comprometedora forma de lucha, en defensa de sus intereses nacionales y en la mayoría de los casos contra un enemigo asimétrico y con un poder mucho mayor, como son los Estados Unidos.

\section{La influencia de China}

Oded Shenkar (2004) en el libro "El Siglo de la China", hace referencia a una serie de prácticas utilizadas por los chinos, que como el contrabando o el hurto de la propiedad intelectual, socavan de alguna forma el statu quo estadounidense en el campo tecnológico, comercial, cultural, entre otros; evidenciando la utilización por parte de China, de nuevos formatos de lucha en los que no existen reglas (Liang et al,1999) y se ataca al adversario de un modo sutil, lento pero sistemático.

De igual manera, Evan Ellis (2014) manifiesta la existencia de un conflicto global, del mismo modo

\footnotetext{
${ }^{4}$ People's Liberation Army por sus siglas en inglés.

${ }^{5}$ Unrestricted Warfare utilizando su traducción al inglés.
}

que lo hace Samuel Huntington (1993) en el que se enfrentan los Estados Unidos y China, y que incluye según Ellis (2014), acciones militares como también devastación mutua con hostilidades en comercio, finanzas, ciberespacio y otras áreas, por lo que las alianzas y capitales comerciales en América Latina y donde sea, se convertirán en herramientas de la lucha entre estos dos poderes.

Así mismo, Robert Kaplan (2010) también habla de la influencia cultural que China ejerce en sus fronteras, particularmente en el Lejano Este Ruso, donde a través de la promoción de la presencia masiva de población en la zona de frontera e incluso traspasando dicha franja y adentrándose hacia Rusia, los chinos logran hacer proyección de poder y gestión de intereses geopolíticos, (Brzezinski, 1997) consolidando de esta forma toda una acción geoestratégica, ya que China tiene la voluntad y la capacidad de ejercer poder en defensa de sus intereses, de forma muy discreta pero a la vez muy efectiva. Lo anterior, sólo es otra expresión, otra forma de lucha de la Guerra Irrestricta señalada en la tesis de este artículo y especialmente aplica para el influjo ejercido por China al competir por preponderancia económica, comercial, cultural, y a futuro, militar con los Estados Unidos, en otros escenarios, como las mismas grandes urbes norteamericanas donde la presencia oriental marca a menudo contrastes y deja ver influencia en diversos índoles.

Analizando las anteriores citas, resultan evidentes los intereses Chinos, que si bien no se expresan abiertamente en su política exterior, ni en su Política Nacional de Defensa del año 2010 (República Popular China, 2010) y de la que se hablará más adelante, las acciones que soportan dichos intereses, saltan a la vista y pretenderían asumir importantes riesgos con el fin de asegurar la supervivencia futura de su Estado (Morgenthau, 1986).

Considérese sólo a manera de ejemplo para justificar estas acciones, el hecho de que Asia tiene el 36\% del agua dulce del mundo, pero contrastantemente, el $60 \%$ de la población mundial. Tal desbalance, se tornaría amenazante frente al resto del mundo, sólo por mencionarlo, Suramérica que tiene el segundo porcentaje más alto del total del agua dulce del mundo, después de Asia, con $28 \%$ y solo un $6 \%$ de la población mundial (Somos amigos de la tierra.org, 2014). En el mismo sentido y en proporciones similares gravita la problemática alimentaria, asociada a la disponibilidad de tierras cultivables en China. Lo anterior, tiene que ver con su explo- 
sión demográfica (Gallois, 1992) y todos los problemas derivados de tal crecimiento de la población, es decir, la dificultad de alimentar y satisfacer las necesidades básicas de un número de personas cada vez más elevado y que tiende a rebasar la capacidad del Estado chino. A esto se debe sumar el tema de la elevada demanda de materias primas y energía, requeridas para satisfacer las voraces necesidades que enfrenta la descomunal producción de la industria china. (Kaplan, 2010, p. 69).

Lo descrito hasta ahora, podría resumirse en lo planteado por un autor chileno al afirmar: "En un contexto global, parece claro que el interés de China en la región no sólo persigue equilibrar sutilmente la influencia de Estados Unidos en su propio hemisferio, de la misma forma que éste país lo hace en Asia, sino, especialmente, asegurarse desde la región el abastecimiento de los recursos naturales que requiere su acelerado crecimiento" (Contreras, 2009, p. 40).

\section{Guerra Irrestricta por parte de China}

Siguiendo los planteamientos anteriores, la principal motivación china es su marcado interés por resurgir como gran imperio y querer disputar la hegemonía global en diversos campos a los Estados Unidos esto es, no sólo en lo económico, también a futuro en lo tecnológico (Shenkar, 2004) lo militar, y lo cultural. Como ya se ha señalado en apartes anteriores, y ¿por qué no pensarlo? en lo político; todo dentro de su lógica milenaria propia, es decir, donde no se considera el factor tiempo como imperativo para el cumplimiento de los objetivos o las metas trazadas. En este sentido, China no tiene prisa en avanzar pero lo hace con paso firme. La misma lógica de la Guerra Popular Prolongada (Rosanía, 2014).

Esta última y principal motivación, amplía la problemática y por supuesto la necesidad de obtener preponderancia global de China, aspirando de este modo a ser la superpotencia hegemónica en el siglo XXI (Kaplan, 2010). Mantener esta meta, requiere de una serie de exigencias constitutivas de colosales retos que se posan sobre los chinos, para lo cual tienen que contemplar su solución a futuro. No en vano, China se encuentra interesada en ampliar y modernizar su marina de guerra, adquiriendo alcance global, para hacer guerras fuera de su espacio vital, defendiendo sus rutas comerciales, de suministros de petróleo y materia prima, (Contreras Polgati, Análisis crítico de la geopolítica contemporánea, 2007) con el poder aéreo embarcado, obteniendo uno o más portaaviones (Kaplan, 2010, p. 73).
Pero para poder lograr todo lo anterior, los chinos deben combinar todas las formas de lucha. Aquí surgiría entonces, el hito de la "Guerra Irrestricta" (Liang et al, 1999) abarcando todas las modalidades de acción posibles, es decir la utilización de todos los medios en contra de un adversario asimétrico, donde las batallas por la victoria implicarán la diversificación en el empleo de medios no letales en los mismos campos de influencia ya descritos. Todo esto, con el ánimo de afectar la voluntad de su gran adversario, antes que el uso de la fuerza militar. No obstante, los chinos también se están preparando en este último campo, como hasta aquí también se ha precisado.

Consecuentemente, un buen ejemplo de combinación de formas de lucha arriba señaladas, se describe en el mismo libro la Guerra Irrestricta:"Cuando la gente comience a inclinarse y regocijarse por la reducción del uso de la fuerza militar para resolver los conflictos, la guerra renacerá en otra forma y en otro ámbito, convirtiéndose en un instrumento de enorme poder en las manos de todos aquellos que alberguen intenciones de controlar otros países o regiones. En este sentido, hay razones para mantener que el ataque financiero de George Soros en el este de Asia, el ataque terrorista contra la embajada de los Estados Unidos por Osama Bin Laden, el ataque con gas en el metro de Tokio por los discípulos de la secta Aum Shinri Kyo, y los estragos causados por personas como Morris Jr. en la Internet, en los cuales los grados de destrucción no son, de ninguna manera, secundarios a los de una guerra, representan una semi-guerra, una cuasi-guerra, una sub-guerra, es decir, la forma embrionaria de otro tipo de guerra." (Liang et al, 1999, p. 4).

En este orden de ideas, pareciera que la doctrina de la Guerra Irrestricta o Guerra sin restricciones, hubiese tenido su origen en el citado escrito del mismo nombre del año 1999, cuyos autores, dos jóvenes coroneles del Ejército chino Qiao Liang y Wang Xiangsui, propusieron cursos de acción para actores que no poseen una gran potencia militar, en particular China, para suplir su debilidad militar frente a los Estados Unidos, en un conflicto de alta tecnología (Liang et al, 1999).

Sin embargo, la incorporación de la doctrina citada en el anterior aparte, al ideario geoestratégico chino, no es nueva y podría leerse entre líneas en obras como la de Sun Tzu (2004) y aunque no se encuentra plasmada explícitamente en documentos rectores oficiales del gobierno chino, autores como Contreras citan su arraigo al interés oriental de la siguiente forma: "Interesante 
resulta, para comprender esta asimétrica complementariedad de objetivos, incursionar en los conceptos de proyección del soft power y en la teoría de la Guerra Irrestricta que forman parte tanto de la política exterior china como de su concepción estratégica de defensa" (Contreras, 2009, p. 40).

Con respecto al concepto de asimetría, (Pombo Celles y Goncalves, 2013) este se manifiesta por ejemplo, de cara a la desproporción evidente entre el costo de los recursos con los que cuenta un hacker informático, ${ }^{6}$ comparado con el costo que implica dotar y mantener organizaciones gubernamentales que se dedican a combatir las amenazas virtuales. (Chang y Granger, 2012). En este punto valdría la pena pensar en el enorme costo que puede tener, toda la Estrategia del Departamento de Defensa para la Operación en el Ciberespacio en los Estados Unidos. (Departamento de Defensa de los Estados Unidos, 2011) El concepto de asimetría, también se puede asimilar si se analiza la guerra sostenida por Mao Tze Tung con el Ejército Japonés y con el Ejército Chino. Es la estrategia del débil contra el fuerte.

Otro punto son los principios de la Guerra Irrestricta (Contreras, 2011), donde se puede destacar la omnidireccionalidad, que se articula al lograr la capacidad de imaginar integralmente toda la situación en prospectiva y de esta manera coordinar el empleo de todos los medios posibles, lo cual da lógica a la utilización de esta doctrina por parte de China.

Del mismo modo, la sincronía, las medidas sin límites, el consumo mínimo, la coordinación multidimensional entre otros principios, son tenidos en cuenta dentro de la estructuración de esta forma de enfrentar enemigos asimétricos.

\section{La ciberguerra china}

En particular, las medidas sin límites, la coordinación multidimensional, se podrían ilustrar por ejemplo con la explotación de los medios de comunicación a favor, para influir en la opinión pública a nivel nacional e internacional, ya que se está usando un campo totalmente diferente y fuera de la órbita de lo armado o letal,7 mientras que también se conjugan acciones desde distintas

\footnotetext{
${ }^{6}$ Se coloca este ejemplo, para comenzar a abordar el tema de la ciberguerra, en atención a lo planteado al inicio del escrito, es decir, considerando a la ciberguerra como una de las principales formas de lucha adoptada por los chinos en el uso de la Guerra Irrestricta.

${ }^{7}$ Medidas sin límites.
}

dimensiones diferentes a lo militar, ${ }_{1}^{8}$ pero que en últimas se tornan en armas estratégicas clave, por supuesto, no letales en el sentido que no provocan la muerte física del adversario, pero si debilitan su voluntad de lucha y su actitud ofensiva.

Vale la pena decir que en la ciberguerra o la utilización del ciberespacio como nueva forma de lucha, el hecho de cuantificar el valor de un ataque cibernético, valor que debe considerarse esencialmente bajo, puede utilizarse como una forma de materializar el principio de consumo mínimo.

Después de todo, en el uso de la ciberguerra como opción derivada de la Guerra Irrestricta, tampoco hay letalidad que pueda provocar muertes físicas, pero si devastación de recursos de comando y control, logísticos, administrativos y por supuesto de organización, planeación y ejecución operativa.

De igual importancia, el aplicar una acción ofensiva en el ciberespacio en el momento indicado, es decir, cuando el enemigo menos lo espere y cuando más vulnerable se encuentre o más le afecte, graficaría el principio restante de la Guerra Irrestricta que no se había ejemplificado hasta ahora, como es el de la sincronía.

Así, la ciberguerra desde la lógica de la Guerra Irrestricta ya no descansa en la fuerza bélica para someter la voluntad del enemigo o adversario, sino que utilizando el concepto de armas amigables o "armas más amables" y"nuevo concepto de armas" (Liang et al, 1999, pp. 15-18) y en general todos los medios necesarios y disponibles que se puedan utilizar en el mundo virtual; defiende los propios intereses, amplia zonas de influencia, se apropia de información o activos informáticos, interrumpe, bloquea o impide el uso del recurso informático y el ciberespacio al enemigo para, en el mejor de los escenarios, obligar al contrincante a aceptar los propios intereses.

Luego, un colapso de internet, un corte de suministro energético provocado por la inserción de un virus en plantas de producción de energía, el colapso en una planta nuclear, otra vez, por la afectación de sus recursos cibernéticos, una crisis económica generada por la manipulación de herramientas informáticas, o el robo de propiedad intelectual a compañías norteamericanas, tal como se marcó en los inicios de este escrito, son situaciones que pueden generar alto costo y daño a la pobla-

${ }^{8}$ Coordinación multidimensional. 
ción. El cambio de concepto frente a la guerra convencional es sustancial, con potenciales consecuencias en todo ámbito, especialmente en el ético, puesto que no existen fronteras en la persecución de los intereses y el punto crítico está en la posibilidad de realizar actos de guerra que causen gran daño de forma soterrada. Cambia así la expresión de la guerra, cambia su naturaleza esencialmente militar como ya se ha descrito, se reitera: es la guerra del débil contra el fuerte.

Considerando que: "La seguridad no formaba parte del diseño original del ciberespacio" (Chang et al, 2012) los chinos han aprovechado esta vulnerabilidad para atacar, socavar, bloquear, restringir, entre otros, el uso de los recursos informáticos y del ciberespacio por parte de los Estados Unidos, sacando de esto grandes réditos como lo son el poderse hacer a información privilegiada, restringida y propiedad intelectual, lo que también se considera espionaje cibernético, con la gran ventaja de que tales acciones por su grado de complejidad, es casi imposible demostrar con exactitud de donde vinieron, luego no es posible reclamar directamente por su autoría en la arena internacional a ningún actor nacional o no nacional, tal y como se reclama y se toman represalias en algunos casos, por las acciones convencionales de guerra como presiones, actos hostiles, ataques o agresiones armadas directas o indirectas.

Hay que destacar, que la doctrina rectora de las operaciones cibernéticas chinas, proviene de los escritos de oficiales del PLA a fines de la década de los noventa, como bien se ha señalado a lo largo de este artículo, y posteriormente estructurada dentro de la estrategia INEW ${ }^{9}$ que concuerdan con compromisos de ataque y de espionaje cibernético con organizaciones que llevan a cabo operaciones similares en el ámbito de la guerra electrónica. Muchos de los "hackers" se han convertido en empresas asesoras o contratistas del gobierno chino o actúan en el contorno académico. La academia china también parece estar hondamente implicada con la guerra cibernética, no tan solo en las investigaciones sino también posiblemente con las operaciones (DeWeese, Krekel, Bakos, y Barnett, 2009).

Entonces, es pertinente revisar lo ocurrido en la operación Aurora, en la cual varias empresas de origen norteamericano como Google, Adobe, Dow Chemical, Northrop Grumman, Symantec y Yahoo entre otras 32 ${ }^{9}$ Estrategia general de la guerra de la información china, por sus siglas en
inglés. firmas, fueron blancos de ataque informáticos, espionaje y robo de propiedad intelectual ${ }^{10}$ y donde se pudo precisar que dichos ataques provenían de servidores ubicados en el territorio continental chino.

Enseguida, tales acciones sugieren el uso de sofisticada ingeniería informática de parte de sus ejecutores, los que usaron instrucciones bastante precisas, lo que daría a entender que los hackers tenían acceso a información de inteligencia adicional y mucho más privilegiada sobre los blancos, lo que finalmente se podría interpretar como un soporte de una organización más elaborada como por ejemplo un Estado Nación (Shakarian et al, 2013b).

Así mismo, dicha operación ejecutada presumiblemente por actores chinos entre finales de 2009 y comienzos de 2010, se puede considerar como una de las muchas evidencias de tal iniciativa de ataque y espionaje cibernético, de la ciberguerra (Thomas, 2008) desde la lógica de la guerra sin límites o Guerra Irrestricta contra los Estados Unidos, más aun si se tiene en cuenta que tal rival para los chinos, posee una cultura avanzada, basando muchos de sus campos del poder en la informática y en el uso del ciberespacio, por lo que la ciberguerra sería el curso de acción ideal a seguir, ante tal preponderancia de una civilización centrada en el uso de la información.

En directa concordancia con lo anterior, se puede citar lo que:

las acciones de una nación estado, se podría pensar que el robo de propiedad intelectual es una forma de guerra económica—nivelar el campo de juego tecnológico para reducir la ventaja de la capacidad industrial de una nación adversaria. Evidentemente, esto está en línea con las ideas chinas de Unrestricted Warfare-donde varias formas de guerra informática ocurren constantemente (inclusive durante tiempos de paz) y atacan todos los aspectos del poder de una nación (incluyendo la industria) (Shakarian et al, 2013a; 2013b, p. 80).

Luego, tales acciones constituyen importantes manifestaciones de esa Guerra Irrestricta librada por parte de China, con un énfasis especial en la ciberguerra, en

${ }^{10}$ Principalmente robo de códigos fuente de software diseñado comercialmente e información sobre activistas chinos de derechos humanos. 
contra de los Estados Unidos y llevadas a cabo con relativa impunidad.

Así, de forma ocultada usando un perfil bajo y furtivo, los chinos usan la ciberguerra como una de las puntas de lanza de su geoestrategia, con el propósito pre entendido de que no podrán generar una respuesta directa de parte del hegemón global. Es decir, sin temor a que estas actividades conduzcan a enfrentamientos militares, dada su naturaleza y carácter que hace que exista una gran dificultad para detectar a los autores. En este tipo de operaciones cibernéticas o de ciberguerra, el límite que permite diferenciar entre acción de paz o acción de guerra, se torna bastante tenue o difuso y precisamente esta característica es la que le da su connotación especial a esta variante de la guerra, porque no se sabe quién generó el ataque y a veces ni siquiera se pueden precisar sus efectos o sólo después de mucho tiempo.

Pese a lo anterior, tanto las corporaciones arriba mencionadas como los diplomáticos norteamericanos al más alto nivel, incluida la Secretaria de Estado en su momento, Hilary Clinton, han elevado sus protestas rigurosas contra el gobierno chino como producto de los ataques y del espionaje (Shakarian et al. 2013a, p. 75). Esto dejaría entrever un vínculo más de atribución hacia los chinos en tales acciones, que pudiera no conocerse públicamente por razones de alto nivel de confidencialidad, pero que iría en el mismo sentido de lo planteado a lo largo del artículo, atribuyendo la práctica de la ciberguerra a los orientales en el marco de la Guerra Irrestricta.

Además, existen otros indicios de la práctica de la ciberguerra por parte de China, en contra de sus rivales asimétricos, tales como la existencia al interior del PLA de una unidad especializada en dichas tareas y denominada la Unidad 61398, (Shakarian et al, 2013b, p. 75) responsable de gran parte del espionaje cibernético hacia naciones angloparlantes.

Por lo que se refiere a la Política Nacional de Defensa China, ${ }^{1}$ en este documento publicado en 2010, (República Popular China, 2010) el país asiático reafirma sus políticas en materia de seguridad y reitera que no pretende dominar el mundo, ni realizar una expansión militar, ni ahora ni en el futuro; señala también que la meta y misión de la defensa nacional es salvaguardar la

11 China's National Defense según su traducción al inglés. soberanía, los beneficios del desarrollo y la integridad territorial. En el escrito, el gobierno chino destaca su política de no uso de armas nucleares, insiste en las estrategias nucleares de autodefensa, y rechaza competir con otros países en cuanto a las reservas de armamentos nucleares se refiere.

En el mismo sentido y según el gobierno chino, se insiste en el nuevo concepto de seguridad, el cual consiste en lograr la confianza, los beneficios, la igualdad y la cooperación mutua, así como en resolver las problemáticas regionales y los conflictos internacionales a través de la vía pacíica. Sobre los gastos militares, el escrito explica que con el desarrollo social y económico, el costo de la defensa nacional mantiene un crecimiento moderado.

Contrario a lo ilustrado hasta ahora en este artículo, la Política Nacional de Defensa estipula que China nunca buscará la hegemonía ni adoptará el enfoque de expansión u obtención de ventaja de cualquier género sobre los actores nacionales que conforman la comunidad internacional, ahora ni en el futuro, sin importar cómo se desarrolle su economía. Como se anota, esto discrepa notoriamente con las evidencias, indicios, hechos y datos antes presentados, que apuntan a lo contrario y la conclusión que se puede sacar es que China predica en su mejor ejecución de diplomacia, ciertos postulados en el documento en mención, pero en la práctica la realidad es otra, ya que existen todos los índices para pensar que China usa de manera soterrada, nuevas formas de lucha como la ciberguerra para obtener ventaja sobre sus contendientes, ventajas que serán capitalizadas a futuro dentro de una confrontación cuando sea necesario, dentro de la disputa por la hegemonía global.

De igual importancia, resulta preocupante encontrar en el documento ${ }^{12}$ que el gasto en defensa de China creció en $17,5 \%$ para 2008 , en $18,5 \%$ para 2009 y para 2010 es 7,5\% mayor de lo que era para 2009; por lo que los chinos manifiestan que ha disminuido, aunque la realidad pareciera apuntar hacia otra parte.

Así es que, un capítulo del escrito oficial que habla sobre la aceleración de la informatización, ${ }^{13}$ para significar la prioridad en desarrollar y actualizar en el PLA toda la base tecnológica de equipos y procesos que utilizan la informática y el ciberespacio como insumos

12 Política Nacional de Defensa China.

13 Término acuñado por los mismos chinos. 
y medios fundamentales, deja pensar que existe una gran preocupación y prioridad sobre los temas informáticos y cibernéticos en concordancia con lo ya planteado sobre la voluntad y la capacidad de practicar la ciberguerra como forma de lucha de la Guerra Irrestricta dentro del ideario geoestratégico chino. Todo lo anterior, acompañado de la consecuente primacía en el presupuesto, que apalanca los grandes costos que esto representa.

Por otro lado, tal preocupación por el uso y control del ciberespacio asociados al desarrollo de la informática, también atiende a la concepción de las tradiciones y cultura china, que en efecto han generado un razonamiento militar en cierto sentido muy distinto al de occidente (Sobies, 2003). Respecto a este último punto, un informe al Congreso de los Estados Unidos del año 2002 (Oficina del Secretario de Defensa de los Estados Unidos de Norteamérica, 2002) es categórico en afirmar que uno de los objetivos estratégicos de China es cómo maximizar la "configuración estratégica de poder" conocida como "shi". Lingüistas chinos lo explican como 'la alineación de las fuerzas', la 'inclinación de las cosas' o 'potencial que nace de la disposición' de lo que solamente un estratega experto se puede aprovechar para garantizar la victoria sobre una fuerza superior.

Otra interpretación del shi se podría enfocar en establecer condiciones favorables. Si una nación - Estado logra alcanzar un nivel de shi más elevado que un rival, este último será derrotado fácilmente cuando el conflicto surja, porque cualquier batalla (inclusive de ser necesaria) se llevará a cabo en condiciones sumamente favorables para la primera nación-ya que ésta ha establecido las condiciones favorables mediante el logro del shi (Shakarian et al, 2013a, p. 76).

\section{Conclusiones}

La anterior cita sirve para concluir sobre la hipótesis planteada al iniciar este artículo, en el sentido que los indicios, hechos y datos son bastante claros para declarar, que en efecto la nación china posee dentro de su pensamiento geoestratégico, la convicción de utilizar todas las formas de lucha, dentro de una lógica de Guerra Irrestricta, con el fin de conseguir las condiciones que a futuro garanticen su victoria sobre un adversario superior, pero pretendiendo desde ahora que tales formas de lucha socaven la voluntad de su enemigo. Esto evidentemente con la utilización de una forma de lucha no letal, sofisticada y acorde con la di- námica de gran civilización de su adversario. Tal forma sofisticada de lucha es la ciberguerra.

Así las cosas, la ciberguerra se constituye en un gran baluarte de proyección de fuerza y organización de prioridades, dentro de una lógica postmoderna pero además donde el factor cronológico es irrelevante, como lo ha sido en la cultura milenaria china, donde los cambios han venido en su momento sin necesidad de presionar resultados, simplemente aplicando la constancia como mandato; la inercia sabia de los procesos históricos se encargará del resto. De esa forma los chinos combaten hoy día, gestionando sus intereses geoestratégicos, vía diversas herramientas, eficientes y sofisticadas para derrotar a su Goliat moderno, en este caso esa herramienta que pertenece a la gran caja de alternativas de la Guerra Irrestricta es la ciberguerra.

La lógica del pensamiento estratégico chino que dista bastante de la occidental y en este sentido, la consecución de objetivos nacionales, no tiene premura en el tiempo, así como tampoco se tienen consideraciones éticas o de otra índole, para ejecutar acciones que paulatinamente debiliten al enemigo, sin necesidad de acudir a la fuerza letal como bien lo pregonaba uno de los autores chinos clásicos de la guerra, (Tzu, 2004) creando de este modo y a futuro las condiciones ideales de un enfrentamiento que garantice la victoria decisiva, habiendo hecho previamente un trabajo dedicado, furtivo, soterrado y sistemático que a lo largo del tiempo haya cambiado el balance de fuerza dentro de la confrontación. El poner la balanza a su favor con acciones como las descritas en este artículo, representa el corazón de la lógica estratégica china en contra de su enemigo, en este caso los Estados Unidos.

\section{Referencias}

Brzezinski, Z. (1997). El Gran Tablero Mundial. Washington D.C.: B Books.

Chang, W., y Granger, S. (2012). La Guerra en el Ámbito Cibernetico. Air \& Space PowerJournal, pp. 83-90.

Contreras Polgati, A. (Octubre - Diciembre de 2007). Análisis crítico de la geopolítica contemporánea. Revista Política y Estrategia (108), pp. 29-45.

Contreras Polgati, A. (Octubre de 2009). Dialéctica Ideológica Regional por la Integración y la Cooperación para el Desarrollo y la Seguridad. UNISCI Discussion Papers (21), 40.

Contreras Polgati, A. (Octubre de 2011). Documento de análisis: principios de la Guerra Irrestricta. Principios de la Guerra Irrestricta. Washington D.C.: Colegio Interamericano de Defensa. 
CIENCIA Y PODER AÉREO | Revista Científica de la Escuela de Postgrados de la Fuerza Aérea Colombiana | Vol. 9 | Enero - Diciembre de 2014

Departamento de Defensa de los Estados Unidos. (2011). Estrategia del Departamento de Defensa para la Operación en el Ciberespacio. Washington D.C.: Departamento de Defensa de los Estados Unidos.

DeWeese, S., Krekel, B., Bakos, G., y Barnett, C. (2009). Capability of the People's Republic of China to Conduct of Cyber Warfare and Computer Network Exploitation. Washington D.C.: Northrop Grumman.

Ellis, R. E. (2014). China's Growing Relationship with Latin America and the Caribbean. Air y Space Power Journal, pp. 3-17.

Gallois, P. (1992). Capítulo quinto: Geopolítica y población. En P. Gallois, Los caminos del poder, pp. 103-123. Madrid: Ejército.

Huntington, S. P. (1993). ¿Choque de civilizaciones? Foreign Affairs, en español, pp. 15-30.

Kaplan, R. D. (2010). La geografía del poder chino. Foreing Affairs Latinoamérica, 10 (3), pp. 66-80.

Liang, Q., y Xiangsui, W. (1999). Guerra Irrestricta. Beijing: PLA Literature and Arts Publishing House.

Morgenthau, H. J. (1986). Capítulo1: Una teoría realista de la política internacional. En H. J. Morgenthau, Política entre las Naciones, la lucha por el poder y la paz., pp. 10-26. Buenos Aires: Grupo Editor Latinoamericano, Colección de Estudios Internacionales.

Oficina del Secretario de Defensa de los Estados Unidos de Norteamérica. (2002). Informe al Congreso sobre el Poder Militar de la República Popular China. Washington D.C.: Departamento de Defensa de los Estado Unidos.

Pombo Celles, L. E., y Goncalves, A. (2013). Lecciones Douhetianas Aplicadas al Combate en el Ciberespacio. Air \& Space Power Journal, pp.14-22.

Republica Popular China. (2010). Defensa Nacional de China en 2010. Beijing: República Popular China.

Rosanía, N. (17 de marzo, 2014). La Guerra Popular Prolongada. Notas de Clase, Notas de clase. Bogotá, D.C., Colombia: Escuela Superior de Guerra.

Shakarian, P., Shakarian, J., y Ruef, A. (2013a). El Dragón y la Computadora. En P. Shakarian, J. Shakarian, y A. Ruef, Introduction to Cyber-Warfare: A Multidisciplinary Approach, pp. 75-76. Mongotmery Alabama: Air \& Space Power Journal.

Shakarian, P., Shakarian, J., y Ruef, A. (2013b). Introduction to CyberWarfare: A Multidisciplinary Approach. New York: Syngress.

Shenkar, O. (2004). El Siglo de la China. Bogotá: Norma.

Sobies, E. (1 de marzo, 2003). SANS Institute InfoSec Reading Room. de SANS Institute InfoSec Reading Room. Retrieved from http://www.sans.org/reading-room/whitepapers/warfare/redefining-role-information-warfare-chinese-strategy896 ? show=redefining-role-information-warfare-chinesestrategy-896\&cat=warfare Search el 1 de abril de 2014.

Somos amigos de la tierra.org. (2 de abril, 2014). www.somosamigosdelatierra.org. [En línea] Disponible en http://www.somosamigosdelatierra.org/06_contaminacion/agua/agua4. html Consultado el 2 de abril de 2014.

Thomas, T. (2008). China's Electronic Long-Range Reconnaissance. Military Review, pp. 47-54.

Tzu, S. (2004). El Arte de la Guerra. Buenos Aires: laeditorialvirtual. com.

Para citar este artículo:

Mancera, J. (2014). La ciberguerra china desde la lógica de la guerra irrestricta. Ciencia y Poder Aéreo, Vol. 9 (1). Pp. 89-96

96 | La ciberguerra china desde la lógica de la guerra irrestricta 